\title{
Serum microRNA-205 as a novel biomarker for cervical cancer patients
}

\author{
Quanhui Ma1, Guiping Wan², Shuxia Wang ${ }^{1}$, Wanwei Yang ${ }^{1}$, Jiaming Zhang ${ }^{1}$ and Xiaoming Yao ${ }^{1 *}$
}

\begin{abstract}
Objective: Serum microRNAs (miRNAs) are a novel class of diagnostic and prognostic biomarkers for numerous cancers. However, the level and clinical relevance of circulating miR-205 transcripts in human serum of cervical cancer patients are unclear. The purpose of this study was to determine serum miR-205 levels in cervical cancer patients and explore its association with clinicopathological factors and prognosis.

Methods: Serum miR-205 expression was investigated in 60 cervical cancer patients and 60 healthy normal controls by using real-time PCR. Correlations between miR-205 expression and the clinicopathological features and prognosis of cervical cancer patients were then evaluated. Receiver operating characteristic curves were used to evaluate the sensitivity and specificity of serum miR-205.

Results: Serum miR-205 was significantly upregulated in cervical cancer patients compared with healthy donors $(p<0.01)$, and a high level of miR-205 expression was correlated with poor tumor differentiation $(p=0.009)$, lymph node metastasis $(p=0.015)$ and increased tumor stage $(p=0.001)$. The serum miR-205 level was capable of separating advanced stage from early stage metastatic cervical cancer from non-metastatic samples and poorly differentiated tumors from differentiated tumors with an area under the curve values of $0.74,0.694$ and 0.717 , respectively. The expression of miR-205 was also higher in the cervical cancer tissues compared with the para-carcinoma tissues. In addition, Kaplan-Meier survival analysis showed that cervical cancer patients with high miR-205 expression tended to have shorter overall survival. In multivariate Cox regression analysis, miR-205 was identified as an independent prognostic marker.
\end{abstract}

Conclusions: Serum miR-205, which is upregulated in cervical cancer, represents a predictive biomarker for the prognosis of cervical cancer patients.

Keywords: Cervical cancer, Serum miR-205, Diagnosis, Prognosis, Biomarker

\section{Introduction}

Cervical cancer, one the most commonly malignant tumors, has become a major health problem for women worldwide, particularly in developing countries [1,2]. Cervical cancer develops step-by-step and involves a sequential progression from normal cervical epithelium to preneoplastic cervical intraepithelial neoplasia and then to invasive cervical cancer [3]. Increasing evidences showed that early detection by testing for high-risk human papillomavirus (HPV) and cervical papilloma smears have

\footnotetext{
* Correspondence: chenfang1006@sina.com

'Department of clinical laboratory, Jiangsu Province hospital on Integration of Chinese and Western Medicine, Nanjing University of Traditional Chinese Medicine, Jiangsu Branch of China Academy of Chinese Medical Science, Nanjing 210028, China

Full list of author information is available at the end of the article
}

reduced cervical cancer mortality. However, these methods do not detedted the development of cervical cancer directly [4]. Therefore, new and less invasive biomarkers are needed to improve the detection and prognostic outcome of cervical cancer.

MicroRNAs (miRNAs), small non-coding RNA molecules, play a central role in post-transcriptional gene regulation by binding to a target site in the 3'-UTR of target mRNAs [5]. MiRNAs are involved in pathological and physiological activities [6]. In recent years, the relationship between miRNA and cancer has become a research focus. Tumor initiation and progression is related to altered expression of miRNAs [7]. MiRNAs are not only expressed in cancer tissues but also in sera $[8,9]$. Further investigation confirmed that circulating miRNAs 
can originate from cancer tissues and are highly stable in serum and plasma [10]. As a result, circulating miRNAs have been exploited as a diagnostic tool for early cancer detection, risk assessment and prognosis [11].

MiR-205 acts as an oncogene by modulating the expression of multiple cancer-related target genes [12]. In addition, miR-205 is significantly overexpressed in human cervical cancer tissues and promotes proliferation and migration of cervical cancer cells by targeting CYR61 and CTGF [13]. Circulating miR-205 has been reported as a biomarker for the detection and diagnosis of lung cancer, particularly at its very early stage [14]. However, the clinical significance of circulating miR-205 levels in cervical cancer remains unclear. Thus, the aim of this study was to evaluate whether serum miR-205 was capable of acting as a diagnostic and prognostic biomarker for cervical cancer patients. Here, we report that serum miR-205 is upregulated in cervical cancer and represents a predictive biomarker for the prognosis of cervical cancer patients.

\section{Results}

\section{Higher levels of miR-205 in the serum of cervical cancer} patients

MiR-205 expression was significantly higher in human cervical cancer than in normal tissue, and it also promotes cervical cancer cell proliferation and migration [13]. Therefore, the level of miR-205 was determined in the serum of the cervical cancer patients. The results showed that among the 60 cervical cancer samples analyzed, serum miR-205 was upregulated 5.74-fold in cervical cancer patients compared with the level in healthy donors. Summarized data from all individuals indicated that the relative expression of miR-205 in cervical cancer patients' serum $(1.18 \pm 0.57)$ was significantly higher than that in healthy donors' serum $(0.2 \pm$ 0.12) $(\mathrm{P}<0.01$, Figure 1$)$.

Higher expression of miR-205 in cervical cancer tissues To further demonstrate the role of miR-205 in cervical cancer, its expression was measured in cervical cancer tissues. As shown in Figure 2, the miR-205 expression was significantly increased ( $>3$-fold higher) in cervical cancer tissues $(n=3)$ compared with paracancerous tissues $(\mathrm{n}=3)(\mathrm{p}<0.01)$, which is consistent with an oncogenic role of $\mathrm{miR}-205$.

\section{Correlation of clinicopathological features of cervical cancer with circulating miR205}

To better understand the potential roles of serum miR205 in cervical cancer development and progression, the relationships between miR-205 and various clinical features of cervical cancer were determined. In the present study, the average fold change of miR-205 (miR-205 in

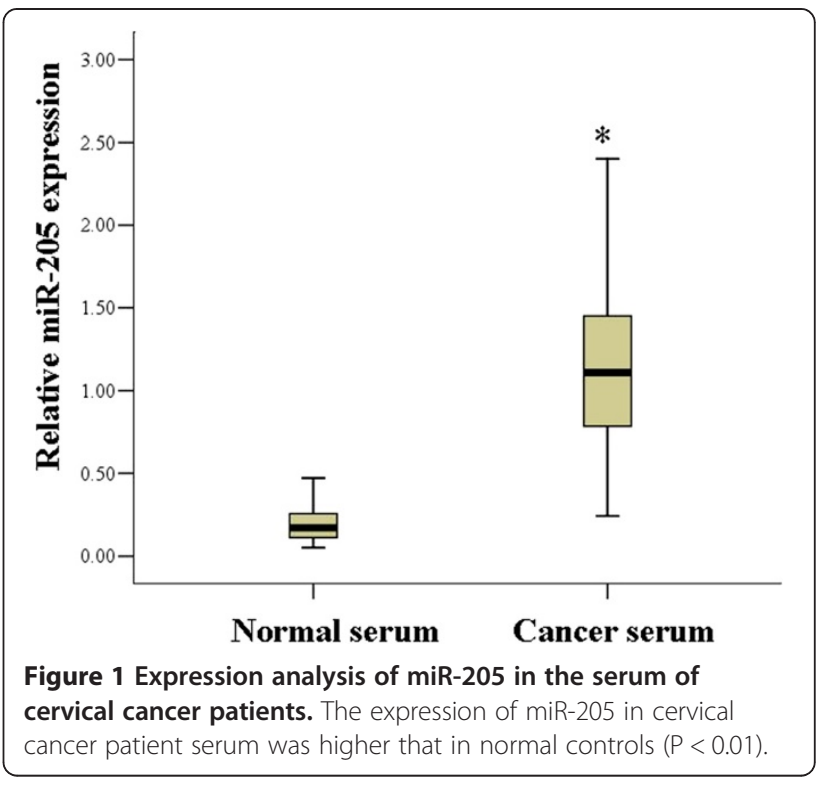

serum of cervical cancer patient compared with healthy donors) was 5.74-fold. The average fold change was used as the threshold, and patients were separated into high expression (above 5.74-fold) and low expression (below 5.74-fold) groups. As shown in Table 1, miR-205 expression was significantly higher in the serum of patients with advanced FIGO stage cervical cancer than those with early FIGO stage ( $\mathrm{P}=0.001$, Table 1$)$. The expression of miR-205 in lymph node metastasis-positive patients was significantly increased compared to that in lymph node metastasis-negative patients $(\mathrm{P}=0.015$, Table 1). There was a tendency for less well differentiated tumors to express higher levels of miR-205 ( $\mathrm{P}=$ 0.009 , Table 1$)$. However, there was no correlation between miR-205 expression and other clinical features, such as age and HPV infection.

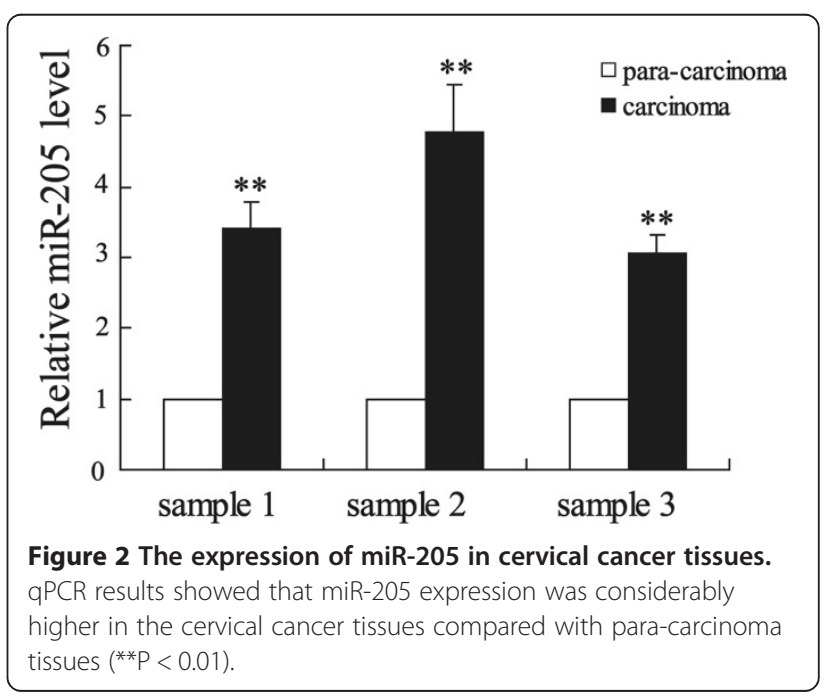


Table 1 Correlation of clinicopathological features of cervical cancer with serum miR-205 expression levels

\begin{tabular}{|c|c|c|c|c|}
\hline \multirow[t]{2}{*}{ Characteristics } & \multirow{2}{*}{$\begin{array}{l}\text { All } \\
\text { cases }\end{array}$} & \multicolumn{3}{|c|}{ Serum miR-205 } \\
\hline & & $\begin{array}{l}\text { High } \\
\text { expression }\end{array}$ & $\begin{array}{l}\text { Low } \\
\text { expression }\end{array}$ & $P$ value \\
\hline Age & & & & 0.796 \\
\hline$<50$ & 28 & 15 & 13 & \\
\hline$\geq 50$ & 32 & 15 & 17 & \\
\hline \multicolumn{5}{|l|}{ Tumor size $(\mathrm{cm})$} \\
\hline$<4$ & 32 & 14 & 18 & 0.438 \\
\hline$\geq 4$ & 28 & 16 & 12 & \\
\hline HPV & & & & 0.671 \\
\hline Positive & 54 & 28 & 26 & \\
\hline Negative & 6 & 2 & 4 & \\
\hline Differentiation & & & & 0.009 \\
\hline $\begin{array}{l}\text { Well-moderately } \\
\text { differentiated }\end{array}$ & 27 & 8 & 19 & \\
\hline Poor differentiation & 33 & 22 & 11 & \\
\hline FIGO stage & & & & 0.001 \\
\hline $\mathrm{lb} \sim \| \mathrm{la}$ & 26 & 6 & 20 & \\
\hline$\|\mathrm{lb} \sim\| \mathrm{lla}$ & 34 & 24 & 10 & \\
\hline Lymph node metastasis & & & & 0.015 \\
\hline No & 22 & 6 & 16 & \\
\hline Yes & 38 & 24 & 14 & \\
\hline
\end{tabular}

\section{Serum miR-205 correlates with prognosis of cervical cancer patients}

To further evaluate whether serum miR-205 levels were associated with cervical cancer prognosis, we performed survival analysis. The survival period of the patients was defined as the duration from the time of surgery to death, or to the last follow-up day. As shown in Figure 3, cervical cancer patients with higher miR-205 expression had significantly poorer survival than those with lower expression of miR-205 (log-rank test: $\mathrm{P}=0.003$ ). The 5year overall survival rate in cervical cancer patients with lower serum miR-205 expression was 53.33\%, whereas that of patients with higher miR-205 expression was $16.67 \%$. To determine the possibility of serum miR-205 as an independent risk factor for poor prognosis, both clinicopathological factors and the level of serum miR205 expression were evaluated by multivariate Cox regression analysis. The results showed that lymph node metastasis and high level of serum miR-205 were independent factors to predict the overall survival of cervical cancer patients (Table 2).

\section{Capability of miR-205 to function as a biomarker for cervical cancer prognosis}

The specificity and sensitivity of miR-205 as a cervical cancer prognosis biomarker was calculated by receiver

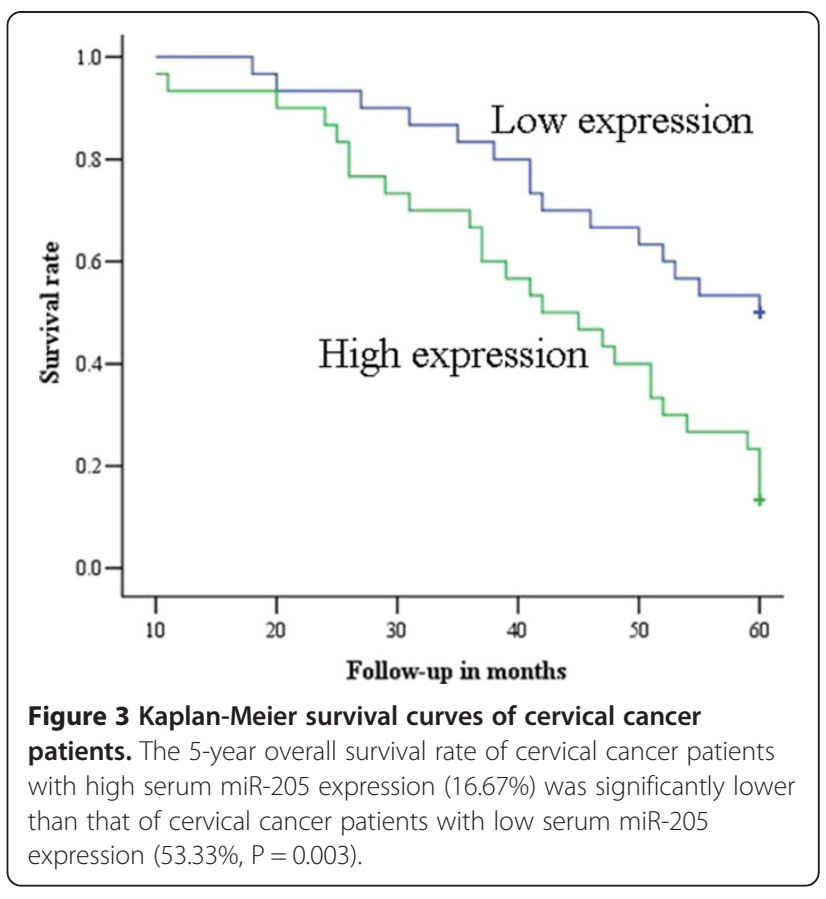

operating characteristic (ROC) curves. ROC analysis revealed that miR-205 had a sensitivity of $76.5 \%$, a specificity of $73.1 \%$ and an area under the curve (AUC) of 0.74 $(\mathrm{p}=0.002$, Figure $4 \mathrm{~A})$ when comparing stages $\mathrm{Ib} \sim \mathrm{IIa}$ and stage IIb IIIa. MiR-205 had an AUC of 0.694 with a sensitivity of $71.1 \%$ and specificity of $72.7 \%(\mathrm{p}=0.013$, Figure 4B) for separation of metastatic cervical cancer from non-metastatic samples. ROC curve analysis also revealed that serum miR-205 was a valuable biomarker to distinguish well to moderately differentiated from poorly differentiation tumors, with a sensitivity of $76.5 \%$, a specificity of $73.1 \%$ and an AUC of 0.717 ( $p=0.004$, Figure 4C). The cut-off value was 0.995 with the highest specificity and sensitivity. Taken together, this analysis revealed that serum miR-205 is a novel and efficient biomarker for cervical cancer prognosis.

\section{Discussion}

Increasing evidence shows that serum miRNAs are promising novel biomarkers for the diagnosis and prognosis of cancer [8]. In this study, we first confirmed by qPCR that miR-205 levels are significantly higher in the serum of cervical cancer patients, and that a high level of miR-205 expression correlated with poor tumor differentiation, lymph node metastasis and increased tumor stage. Notably, patients with high serum miR-205 levels had a significantly lower survival rate than those with low expression levels, and serum miR-205 was an independent risk factor for poor prognosis. These results suggested that serum miR-205 could be used as a potential predictor of prognosis in cervical cancer. 
Table 2 Multivariate analysis for prognostic factors

\begin{tabular}{llll}
\hline Variate & Subset & Relative risk (95\% Cl) & P value \\
\hline Age $($ year) & $<50 / \geq 50$ & $0.661(0.294-1.484)$ & 0.315 \\
Tumor size $(\mathrm{cm})$ & $<4 / \geq 4$ & $1.341(0.616-3.919)$ & 0.460 \\
Tumor differentiation & Well-moderately/poor & $0.836(0.057-12.347)$ & 0.896 \\
FIGO stage & Ib $\sim$ Ila/llb IIla & $0.577(0.038-8.702)$ & 0.691 \\
Lymph node metastasis & Yes/no & $0.032(0.003-0.332)$ & $0.004^{*}$ \\
miR-205 & Low/high & $3.011(1.312-6.910)$ & $0.009^{*}$ \\
\hline
\end{tabular}

$95 \% \mathrm{Cl}: 95 \%$ confidence interval. ${ }^{*} \mathrm{P}<0.05$.

MiR-205 is frequently dysregulated in many cancers and acts as a tumor suppressor or an oncogene depending on cellular context [15]. In cervical cancer, miR-205 functions as an oncogene, promoting proliferation and migration of cancer cells [13]. Our findings are suggestive of an oncogenic role for miR-205, with higher circulating expression of miR-205 in cervical cancer patients with a lower survival rate. In this study, we identified that miR205 expression was significantly increased in cervical cancer tissues compared with paracancerous tissues. In

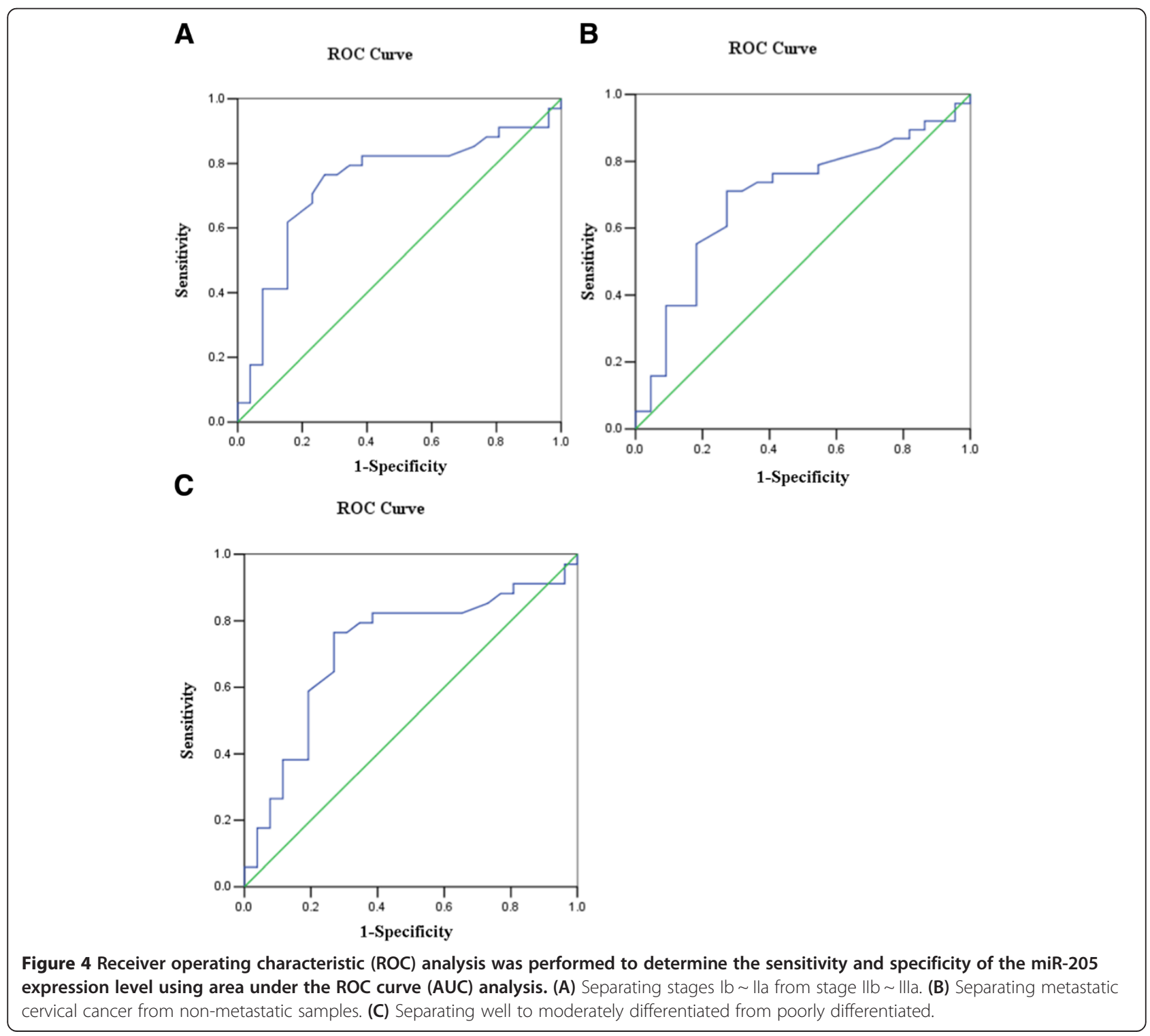


addition, we confirmed, for the first time, that cervical cancer patients had significantly elevated miR-205 levels in their serum samples. This may be explained by miR205 being released from cervical cancer cells into the peripheral blood, which is consistent with previous reports [15]. An increase in serum miR-205 may create a cancerand metastasis-promoting environment. Thus, high levels of miR-205 expression were correlated with poor tumor differentiation, metastasis and increased tumor stage.

Tumor tissue miR-205 or serum miR-205 are associated with the development and prognosis of tumors [15]. Lebanony et al. reported that miR-205 in tissue samples is a highly accurate marker for distinguishing squamous from nonsquamous non-small-cell lung carcinoma [16]. Moreover, the downregulation of miR-205 expression in colorectal cancer tissue could predict the risk of lymph node metastasis [17]. Circulating miR-205 and let- $7 \mathrm{f}$ together are diagnostic biomarkers for ovarian cancer [18]. Aushev et al. revealed that the level of plasma miR-205 strikingly decreased in patients after removal of lung squamous cell carcinoma [19]. We also assessed whether the miR-205 expression level could function as a tumor marker to distinguish advanced stage from early stage, metastatic cervical cancer from non-metastatic samples and poorly differentiated tumors from differentiated tumors. We found serum miR-205 was a valuable biomarker for cervical cancer patients.

Metastatic spread to regional lymph nodes is considered the most important prognostic factor in patients with cervical cancer [20]. Currently, serum miRNAs have been used to identify lymph node metastasis (LNM) in cervical cancer patients. Zhao et al. reported that miR-20a might be a potential biomarker for detecting the lymph node status of cervical cancer patients, with an AUC of 0.734 , a sensitivity of $75 \%$ and a specificity of $72.5 \%$ [21]. The comprehensive set of serum miRNAs (miR-1246, miR-20a, miR-2392, miR-3147, miR-3162-5p and miR-4484) have great potential to serve as potential biomarkers for LNM in early-stage squamous cell carcinoma, with an AUC of 0.992, a sensitivity of 0.967 and a specificity of 0.950 [22]. In this study, our analysis suggested that serum miR-205 might be a novel biomarker for detecting LNM in cervical cancer patients. Our data also revealed that high levels of serum miR-205 and LNM are independent predictors of poor prognosis. Therefore, as a potential prognostic biomarker in cervical cancer, the use of miR-205 might improve patients' risk stratification and guide their treatment.

\section{Conclusions}

We observed that serum miR-205 levels could distinguish patients with cervical cancer from healthy controls. The concentration of circulating miR-205 may be an important blood biomarker for cervical cancer screening and represents a potentially useful biomarker for disease progression. The present data suggested that miR205 might be a valuable circulating marker for cervical cancer with the potential to be translated into clinical applications.

\section{Methods}

Participants

Cervical cancer patients who underwent surgery between January 2008 and August 2012 in Jiangsu Province hospital on Integration of Chinese and Western Medicine were enrolled in the present study. No patients had preoperative chemotherapy, radiotherapy or other treatment history or other inflammatory diseases. The 60 enrolled patients were aged from 34 to 71 years, with a median age of 51 years. The pathological diagnosis of all 60 cervical cancer patients was cervical squamous cell carcinoma. The 60 control subjects were age-matched, healthy volunteers with no current or previous malignancy. All enrolled individuals gave their informed consent, and the Ethical and Scientific Committees of the hospital approved this study. The degree of differentiation was well to moderately differentiated in 27 cases, and poorly differentiated in 33 cases. Thirty-eight cases had lymph node metastases, while 22 cases did not have lymph node metastases. The clinical stage determined was according to the International League of Gynecology and Obstetrics (FIGO, 2009). Twenty-six stage $\mathrm{Ib} \sim$ IIa cases were grouped as early stage, and a total of 34 stage IIb IIIa cases were grouped as late stage. The clinicopathological information of the patients was summarized in Table 1.

\section{Serum collection}

Blood samples were collected in red tiger-top gel separator tubes (Thermo Fisher Scientific Inc., Waltham, MA, USA) from patients or healthy donors. All samples were processed within $2-5 \mathrm{~h}$ after collection as follows: the serum was separated by centrifugation at $1,200 \times \mathrm{g}$ at $4^{\circ} \mathrm{C}$ for $20 \mathrm{~min}$ and passed through a $13-\mathrm{mm}$ serum filter (Thermo Fisher Scientific Inc.). Serum was divided into aliquots and flash frozen at $-80^{\circ} \mathrm{C}$ until total RNA isolation.

\section{Tissue samples}

The non-tumor counterparts were obtained from a section of the resected specimen at the farthest distance from tumor $(>2 \mathrm{~cm}$ from the tumor). Tumor and adjacent non-tumor samples were collected at the time of the curative surgery. Resected specimens were routinely processed for histopathological assessment. Each cervical tissue sample (approximately $100 \mathrm{mg}$ ) was used for microRNA measurement. 


\section{MiRNA extraction and Quantitative real-time polymerase chain reaction (qPCR)}

The Trizol reagent (Invitrogen, Carlsbad, CA, USA) was used to extract total RNA from tissue, according to the manufacturer's instructions. The miRNeasy Serum/ Plasma Kit (Qiagen, Valencia, CA, USA) was used to purify RNA from serum samples according to the manufacture's instructions. The yields of total RNA were 150$300 \mathrm{ng}$ per $400 \mu \mathrm{l}$ of serum. For qPCR, miRNA-specific TaqMan MicroRNA Assays (Applied Biosystems, Foster City, CA, USA) for miR-16 (reference miR for serum), U6 (reference miR for tissue) and miR-205 were performed, as described by the manufacturer. Briefly, $100 \mathrm{ng}$ of total RNA was reverse transcribed using primers specific to each miRNA target, followed by realtime PCR using an Applied Biosystems 7000 Sequence Detection System.

The average expression levels of miR-205 were normalized against miR-16 or U6 using the $2^{-\Delta \mathrm{Ct}}$ method. Differences between the groups were presented as $\Delta \mathrm{Ct}$, indicating the difference between the $\mathrm{Ct}$ value of miR205 and the Ct value of miR-16 or U6. To ensure consistent measurements throughout all assays, for each PCR amplification reaction, three independent RNA samples were loaded as internal controls to account for any plate-to-plate variation, and the results from each plate were normalized against internal normalization controls.

\section{Statistical analyses}

Statistical analyses were performed using statistical analysis software SPSS 13.0. Data were expressed as the mean \pm SD. Analysis of variance (ANOVA) was used to determine the statistical differences among the groups. The Kaplan-Meier method was used to estimate survival rates. A multivariate analysis of the independent prognostic factors was conducted using the Cox proportional hazards model. A receiver operating characteristic (ROC) curve was also constructed to evaluate the specificity and sensitivity of distinguishing advanced stage from early stage, metastatic cervical cancer from nonmetastatic samples, and poorly differentiated tumors from differentiated tumors by miR-205 expression levels. A $P$ value $<0.05$ was deemed significant. A $P$ value $<$ 0.01 was deemed highly significant.

\section{Competing interests}

The authors declare that they have no competing interests.

\section{Authors' contributions}

QM carried out the molecular genetic studies and provided conception and design. GW and SW participated in data acquisition. WY participated in the sequence alignment. JZ contributed to statistical analysis. XY drafted the manuscript and participated in data analysis and interpretation. All authors read and approved the final manuscript.

\section{Author details}

'Department of clinical laboratory, Jiangsu Province hospital on Integration of Chinese and Western Medicine, Nanjing University of Traditional Chinese Medicine, Jiangsu Branch of China Academy of Chinese Medical Science, Nanjing 210028, China. ${ }^{2}$ Department of obstetrics and gynecology, Jiangsu Province Hospital on Integration of Chinese and Western Medicine, Nanjing, China.

Received: 19 May 2014 Accepted: 1 August 2014

Published: 22 August 2014

\section{References}

1. Wenzel L, Dogan-Ates A, Habbal R, Berkowitz R, Goldstein DP, Bernstein M, Kluhsman BC, Osann K, Newlands E, Seckl MJ, Hancock B, Cella D: Defining and measuring reproductive concerns of female cancer survivors. J Natl Cancer Inst Monogr 2005, 34:94-98.

2. Paradkar PH, Joshi JV, Mertia PN, Agashe SV, Vaidya RA: Role of cytokines in genesis, progression and prognosis of cervical cancer. Asian Pac J Cancer Prev 2014, 15:3851-3864.

3. Mariuzzi G, Santinelli A, Valli M, Sisti S, Montironi R, Mariuzzi L, Alberti R, Pisani E: Cytometric evidence that cervical intraepithelial neoplasia I and II are dysplasias rather than true neoplasias. An image analysis study of factors involved in the progression of cervicallesions. Anal Quant Cytol Histol 1992, 14:137-147.

4. Behtash N, Mehrdad N: Cervical cancer: screening and prevention. Asian Pac J Cancer Prev 2006, 7:683-686.

5. Bartel DP: MicroRNAs: genomics, biogenesis, mechanism, and function. Cell 2004, 116:281-297.

6. Williams AE: Functional aspects of animal microRNAs. Cell Mol Life Sci 2008, 65:545-562.

7. Ruan K, Fang X, Ouyang G: MicroRNAs: novel regulators in the hallmarks of human cancer. Cancer Lett 2009, 285:116-126.

8. Allegra A, Alonci A, Campo S, Penna G, Petrungaro A, Gerace D, Musolino C: Circulating microRNAs: new biomarkers in diagnosis, prognosis and treatment of cancer. Int J Oncol 2012, 41:1897-1912.

9. Anindo $\mathrm{Ml}$, Yaqinuddin $\mathrm{A}$ : Insights into the potential use of microRNAs as biomarker in cancer. Int J Surg 2012, 10:443-449.

10. Mitchell PS, Parkin RK, Kroh EM, Fritz BR, Wyman SK, Pogosova-Agadjanyan EL, Peterson A, Noteboom J, O'Briant KC, Allen A, Lin DW, Urban N, Drescher CW, Knudsen BS, Stirewalt DL, Gentleman R, Vessella RL, Nelson PS, Martin $D B$, Tewari M: Circulating microRNAs as stable blood-based markers for cancer detection. Proc Natl Acad Sci U S A 2008, 105:10513-10518.

11. Wittmann J, Jäck HM: Serum microRNAs as powerful cancer biomarkers. Biochim Biophys Acta 1806, 2010:200-207.

12. Wang X, Tang S, Le SY, Lu R, Rader JS, Meyers C, Zheng ZM: Aberrant expression of oncogenic and tumor-suppressive microRNAs in cervical cancer is required for cancer cell growth. PLoS One 2008, 3:e2557.

13. Xie H, Zhao Y, Caramuta S, Larsson C, Lui WO: miR-205 expression promotes cell proliferation and migration of human cervical cancer cells. PLoS One 2012, 7:e46990.

14. Lebanony D, Benjamin H, Gilad S, Ezagouri M, Dov A, Ashkenazi K, Gefen N, Izraeli S, Rechavi G, Pass H, Nonaka D, Li J, Spector Y, Rosenfeld N, Chajut A, Cohen D, Aharonov R, Mansukhani M: Diagnostic assay based on hsa-miR-205 expression distinguishes squamous from nonsquamous non-small-cell lung carcinoma. J Clin Oncol 2009, 27:2030-2037.

15. Qin AY, Zhang XW, Liu L, Yu JP, Li H, Wang SZ, Ren XB, Cao S: MiR-205 in cancer: an angel or a devil? Eur J Cell Biol 2013, 92:54-60.

16. Moldovan L, Batte K, Wang Y, Wisler J, Piper M: Analyzing the circulating microRNAs in exosomes/extracellular vesicles from serum or plasma by qRT-PCR. Methods Mol Biol 2013, 1024:129-145.

17. Orang AV, Safaralizadeh R, Hosseinpour Feizi MA, Somi MH: Diagnostic and prognostic value of miR-205 in colorectal cancer. Asian Pac J Cancer Prev 2014, 15:4033-4037

18. Zheng $H$, Zhang L, Zhao Y, Yang D, Song F, Wen Y, Hao Q, Hu Z, Zhang W, Chen K: Plasma miRNAs as diagnostic and prognostic biomarkers for ovarian cancer. PLoS One 2013, 8:e77853.

19. Lebanony D, Benjamin H, Gilad S, Ezagouri M, Dov A, Ashkenazi K, Gefen N, Izraeli S, Rechavi G, Pass H, Nonaka D, Li J, Spector Y, Rosenfeld N, Chajut A, Cohen D, Aharonov R, Mansukhani M: Diagnostic assay based on hsa-miR-205 expression distinguishes squamous from nonsquamous non-small-cell lung carcinoma. J Clin Oncol 2009, 27:2030-2037. 
20. Lukaszuk K, Liss J, Wozniak I, Sliwinski W, Emerich J, Wojcikowski C: HPV and histological status of pelvic lymph node metastases in cervical cancer: a prospective study. J Clin Pathol 2004, 57:472-476.

21. Zhao S, Yao D, Chen J, Ding N: Circulating miRNA-20a and miRNA-203 for screening lymph node metastasis in early stage cervical cancer. Genet Test Mol Biomarkers 2013, 17:631-636.

22. Chen J, Yao D, Li Y, Chen H, He C, Ding N, Lu Y, Ou T, Zhao S, Li L, Long F: Serum microRNA expression levels can predict lymph node metastasis in patients with early-stage cervical squamous cell carcinoma. Int I Mol Med 2013, 32:557-567.

doi:10.1186/s12935-014-0081-0

Cite this article as: Ma et al:: Serum microRNA-205 as a novel biomarker for cervical cancer patients. Cancer Cell International 2014 14:81.

\section{Submit your next manuscript to BioMed Central and take full advantage of:}

- Convenient online submission

- Thorough peer review

- No space constraints or color figure charges

- Immediate publication on acceptance

- Inclusion in PubMed, CAS, Scopus and Google Scholar

- Research which is freely available for redistribution 